\title{
Publisher Correction: Inhibitory modulation of cytochrome $c$ oxidase activity with specific near-infrared light wavelengths attenuates brain ischemia/ reperfusion injury
}

Thomas H. Sanderson $\mathbb{1}^{1,2,3,4}$, Joseph M. Wider ${ }^{2,4,5}$, Icksoo Lee ${ }^{6,7}$, Christian A. Reynolds ${ }^{1,4,5}$, Jenney Liu ${ }^{6}$, Bradley Lepore ${ }^{1}$, Reneé Tousignant ${ }^{1}$, Melissa J. Bukowski ${ }^{1,5}$, Hollie Johnston ${ }^{6}$, Alemu Fite ${ }^{6}$, Sarita Raghunayakula ${ }^{1,2}$, John Kamholz ${ }^{6}$, Lawrence I. Grossman ${ }^{6,4}$, Karin Przyklenk ${ }^{1,4,5}$ \& Maik Hüttemann ${ }^{6,4}$

Correction to: Scientific Reports https://doi.org/10.1038/s41598-018-21869-x, published online 22 February 2018 In the original version of this Article, Affiliations 1,2 and 3 were not listed in the correct order. The correct affiliations are listed below:

Affiliation 1:

Department of Emergency Medicine, Wayne State University School of Medicine, Detroit, MI, 48201, USA. Affiliation 2:

Department of Emergency Medicine, University of Michigan Medical School, Ann Arbor, MI, 48109, USA. Affiliation 3:

Department of Molecular and Integrative Physiology, University of Michigan Medical School, Ann Arbor, MI, 48109, USA.

This error has now been corrected in the PDF and HTML versions of the Article.

\footnotetext{
${ }^{1}$ Department of Emergency Medicine, Wayne State University School of Medicine, Detroit, MI, 48201, USA. ${ }^{2}$ Department of Emergency Medicine, University of Michigan Medical School, Ann Arbor, MI, 48109, USA. ${ }^{3}$ Department of Molecular and Integrative Physiology, University of Michigan Medical School, Ann Arbor, MI, 48109, USA. ${ }^{4}$ Cardiovascular Research Institute, Wayne State University School of Medicine, Detroit, MI, 48201, USA. ${ }^{5}$ Department of Physiology, Wayne State University School of Medicine, Detroit, MI, 48201, USA. ${ }^{6}$ Center for Molecular Medicine and Genetics, Wayne State University School of Medicine, Detroit, MI, 48201, USA. ${ }^{7}$ College of Medicine, Dankook University, Cheonan-si, Chungcheongnam-do, 31116, Republic of Korea. Correspondence and requests for materials should be addressed toT.H.S. (email: thsand@med.umich.edu) or M.H. (email: mhuttema@ med.wayne.edu)
} 
(i) Open Access This article is licensed under a Creative Commons Attribution 4.0 International License, which permits use, sharing, adaptation, distribution and reproduction in any medium or format, as long as you give appropriate credit to the original author(s) and the source, provide a link to the Creative Commons license, and indicate if changes were made. The images or other third party material in this article are included in the article's Creative Commons license, unless indicated otherwise in a credit line to the material. If material is not included in the article's Creative Commons license and your intended use is not permitted by statutory regulation or exceeds the permitted use, you will need to obtain permission directly from the copyright holder. To view a copy of this license, visit http://creativecommons.org/licenses/by/4.0/.

(C) The Author(s) 2018 